DOI:

10.1038/nri2175

\title{
AUTOIMMUNITY
}

\section{Controlling promiscuity}

The promiscuous expression of peripheral tissue antigens in the thymus has been heralded as a means to tolerize or eliminate developing $\mathrm{T}$ cells that are specific for such self antigens before they reach the periphery. It therefore provides protection from organspecific autoimmune diseases. However, besides an established key role for the transcriptional regulator AIRE (autoimmune regulator), the mechanism of promiscuous expression by thymic epithelial cells (TECs) is ill-defined. Now, a study in Nature describes a mechanism for controlling the thymic expression of a tissue-restricted antigen that is the focus of autoreactivity in myasthenia gravis and shows that patients with early onset of this disease are more likely to have a variant of the autoantigen gene that fails to be promiscuously expressed.

To determine whether genetic variation in the prototypic autoantigen in myasthenia gravis, CHRNA1. which encodes the $\alpha$-subunit of the muscle acetylcholine receptor, influences thymic expression or susceptibility to the disease, the authors scanned the CHRNA1 locus for polymorphisms. Of the 70 variants

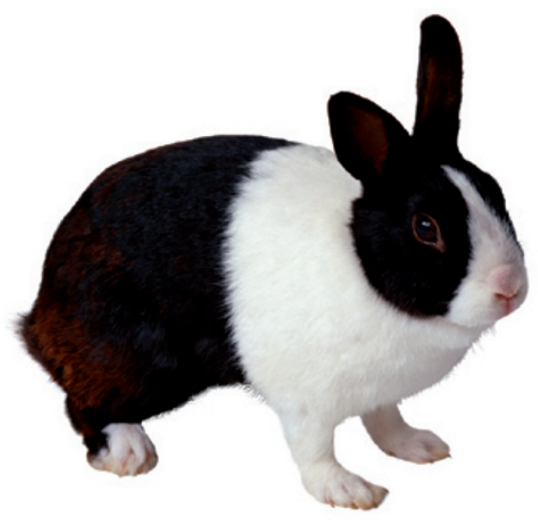

identified, one set of polymorphisms seemed to be more common in patients with early onset of myasthenia gravis. Further analysis of this set of polymorphisms revealed that one single nucleotide polymorphism (converting an adenine to a guanine) present in the promoter for CHRNA1 disrupted a consensus binding site for interferon-regulatory factor 8 (IRF8). Importantly, the presence of the $\mathrm{G}$ allele of CHRNA1 was associated with earlier onset of disease in two independent patient cohorts.

So, does this genetic variation have functional significance? A gel shift assay showed that IRF8 only bound to the radiolabelled oligonucleotide probe corresponding to the A allele promoter of CHRNA1 and not to the $\mathrm{G}$ allele promoter, confirming that this polymorphism disrupts the IRF8-binding site. Studies of a macrophage cell line and a TEC line transfected with reporter-gene constructs containing either the $\mathrm{A}$ allele or $\mathrm{G}$ allele CHRNA1 promoter showed that the $\mathrm{G}$ allele promoter failed to upregulate reporter-gene expression in an IRF8dependent manner. Finally, using chromatin immunoprecipitation they showed that, in TECs, IRF8 binds to

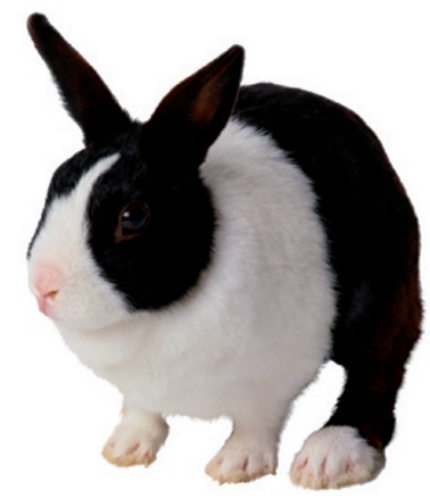

the A allele CHRNA1 promoter and enhances its transcriptional activity.

Given that AIRE is known to be involved in regulating promiscuous gene expression in TECs, the authors next tested for an influence of AIRE on CHRNA1 expression. Although the levels of AIRE and CHRNA1 mRNA in medullary TECs purified from healthy human thymi varied among individuals, there was a close correlation between the expression levels of these two genes. This was not the case, however, in TECs from individuals carrying the $\mathrm{G}$ allele CHRNA1 promoter; TECs from these individuals had approximately twofold lower levels of CHRNA1 mRNA than individuals who were homozygous for the A allele. A direct relationship between AIRE and CHRNA1 expression was confirmed by co-transfection studies, which indicated that the presence of AIRE increases transcription twice as much from the A allele as from the $\mathrm{G}$ allele promoter.

So, based on their observations, the authors propose that AIRE together with IRF8 might constitute part of a transcriptional complex that regulates peripheral-tissue gene expression in the thymus. By disrupting IRF8 binding to the CHRNA1 promoter, the minor $\mathrm{G}$ allele would also affect the co-transcriptional activity of AIRE and reduce CHRNA1 thymic expression, which in turn might modify susceptibility to myasthenia gravis. 